\title{
Birational splitting and algebraic group actions
}

\author{
Vladimir L. Popov ${ }^{1,2}$
}

Received: 22 December 2014 / Revised: 25 April 2015 / Accepted: 28 April 2015 /

Published online: 16 May 2015

(C) Springer International Publishing AG 2015

\begin{abstract}
According to a classical theorem, every algebraic variety endowed with a nontrivial rational action of a connected linear algebraic group is birationally isomorphic to a product of another algebraic variety and $\mathbf{P}^{s}$ with strictly positive $s$. We show that the classical proof of this theorem actually works only in characteristic 0 and we give a characteristic free proof of it. To this end we prove and use a characterization of connected linear algebraic groups $G$ with the property that every rational action of $G$ on an irreducible algebraic variety is birationally equivalent to a regular action of $G$ on an affine algebraic variety.
\end{abstract}

Keywords Algebraic group $\cdot$ Algebraic variety $\cdot$ Action $\cdot$ Rational quotient

Mathematics Subject Classification 20B27

1. Throughout this note $k$ stands for an algebraically closed field of arbitrary characteristic which serves as domain of definition for each of the algebraic varieties considered below. Each algebraic variety is identified with its set of $k$-rational points. We use freely the standard notation and conventions of $[7,11]$ and refer to [6-10] regarding the definitions and basic properties of rational and regular (morphic) actions of algebraic

Supported by Grants RFBR 15-01-02158 and NSh-2998.2014.1.

$凶$ Vladimir L. Popov

vpopov03@gmail.com; popovvl@mi.ras.ru

1 Steklov Mathematical Institute, Russian Academy of Sciences, Gubkina 8, Moscow 119991, Russia

2 Higher School of Economics, National Research University, Myasnitskaya 20, Moscow 101000, Russia 
groups on algebraic varieties. Given a rational action of such a group $G$ on an irreducible algebraic variety $X$, we denote by $\pi_{G, X}: X \rightarrow X_{\prime}^{\prime} G$ a rational quotient of this action; the latter means that $X, G$ and $\pi_{G, X}$ are respectively an irreducible variety and a dominant rational map such that $\pi_{G, X}^{*}\left(k\left(X_{i}^{\prime} G\right)\right)=k(X)^{G}$.

2. Up to a change of notation and terminology, the following statement appeared in the classical paper [4, Theorem 1].

Theorem 1 Assume that a connected linear algebraic group $G$ acts rationally and nontrivially on an irreducible algebraic variety $X$, and let $B$ be a Borel subgroup of $G$. Then $X$ is birationally isomorphic to $\mathbf{P}^{s} \times X, B$, where $\pi_{B, X}: X \rightarrow X_{i}^{\prime} B$ is a rational quotient of the natural rational action of $B$ on $X$ and $0<s \leqslant \operatorname{dim} B$.

In [4] no restriction on char $k$ is imposed, but actually the brief argument given there in support of Theorem 1 works only if char $k=0$. We reproduce it below in order to pinpoint where the restriction char $k=0$ is implicitly used.

Proof (Argument from [4] supporting Theorem 1) Since $G$ is generated by its Borel subgroups and since all Borel subgroups are conjugate to each other, $B$ acts on $X$ nontrivially. Since $B$ is a connected solvable linear algebraic group, there is a chain of connected subgroups

$$
B=B_{0} \supset B_{1} \supset \cdots \supset B_{n}=\{e\}
$$

such that all $B_{i}$ are normal in $B$ and $\operatorname{dim} B_{i}=\operatorname{dim} B-i$. If $d$ is the largest index $i$ such that the action of $B_{i}$ on $X$ is not trivial, then let

$$
\pi_{B_{d}, X}: X \rightarrow X_{i}^{\prime} B_{d} \stackrel{\text { def }}{=} X_{d}
$$

be a rational quotient of $X$ with respect to $B_{d}$. By the cross-section theorem [8, Theorem 10] we find that $X$ is birationally equivalent to $\mathbf{P}^{1} \times X_{d}$. The factor group $B / B_{d}$ acts on $X_{d}$ and we can repeat the same argument.

3. The assumption char $k=0$ is actually implicitly used in the penultimate phrase of this argument. Indeed, it purports the following. Consider a section of $\pi_{B_{d}, X}$, i.e., a rational map $\sigma: X_{d} \rightarrow X$ such that $\pi_{B_{d}, X} \circ \sigma=$ id. Since $B_{d+1}$ lies in the kernel of the action of $B_{d}$ on $X$, this action is reduced to that of the one-dimensional connected linear algebraic group $B_{d} / B_{d+1}$. This action is nontrivial, hence the $B_{d} / B_{d+1}$-stabilizers of points of a dense open subset of $X$ are finite; in particular, the kernel $K$ of this action is finite. The action of $C_{d}=\left(B_{d} / B_{d+1}\right) / K$ on $X$ is faithful, and (1) is its rational quotient.

Being a connected one-dimensional linear algebraic group, $C_{d}$ is isomorphic to either $k^{\times}$(the multiplicative group of $k$ ) or $k^{+}$(the additive group of $k$ ); see, e.g., [11, Theorem 3.4.9].

If it is isomorphic to $k^{\times}$, then faithfulness of its action on $X$ implies that this action is locally free (i.e., $C_{d}$-stabilizers of points of a dense open subset of $X$ are trivial); see [6, Lemma 2.4]. Therefore, the dominant rational map $\gamma: C_{d} \times X_{d}--\rightarrow X$, 
$(c, b) \mapsto c \cdot \sigma(b)$, is bijective over a dense open subset of $X$. If char $k=0$, the latter implies that $\gamma$ is a birational isomorphism and hence $X$ is birationally isomorphic to $\mathbf{P}^{1} \times X_{d}$ because the group variety of $C_{d}$ is rational. But if char $k>0$, one can only say that $\gamma$ is either a birational isomorphism or purely inseparable. The following example shows that the latter indeed may occur.

Example 1 Let char $k=p>0$. Consider the locally free action of $G=B=k^{\times}$ on $X=k \backslash\{0\}$, given by $b \cdot x=b^{p} x$. We have $n=1, d=0, C_{0}=G$, and $X_{0}$ is a point. Therefore, $C_{0} \times X_{0}$ is naturally identified with $G$. Let $\sigma$ map $X_{0}$ to 1 . Then $\gamma^{*}(k(X))=k\left(t^{p}\right) \varsubsetneqq k(t)=k(G)$, where $t$ is the standard coordinate function on $G$. Thus $\gamma$ is not a birational isomorphism.

If $C_{d}$ is isomorphic to $k^{+}$, the same argument works if we know that the action of $C_{d}$ on $X$ is locally free. If char $k=0$, then local freeness indeed holds because in this case there are no nontrivial finite subgroups in $k^{+}$. However, if char $k>0$, it may happen that the action of $C_{d}$ on $X$ is not locally free; therefore, $\gamma$ is not bijective over a dense open subset of $X$, and a fortiori is not a birational isomorphism. The example below is a generalization of the one the author first learned from G. Kemper, whom we thank for it. It is based on the idea going back to [7, 7.1, Example $\left.1^{\circ}\right]$ and Corollary of Proposition 1 below.

Example 2 Let char $k=p>0$ and let $G=B=k^{+}$. Let $x \in k[G]$ be the standard coordinate function on $G$. Every homomorphism of algebraic groups $f: G \rightarrow G$ defines a regular action of $G$ on $X=k^{2}$ by the formula

$$
u \cdot(a, b)=(a+u b+f(u), b), \quad u \in G, \quad(a, b) \in X .
$$

From (2) we infer that an element $u \in G$ lies in the $G$-stabilizer of a point $(a, b) \in X$ if and only if $u$ is a root of the polynomial $f(x)+b x$. By [11, Lemma 3.3.5] there are nonzero elements $\alpha_{1}, \ldots, \alpha_{s} \in k$ and an increasing sequence of nonnegative integers $n_{1}, \ldots, n_{s}$ such that

$$
f=\alpha_{1} x^{p^{n_{1}}}+\cdots+\alpha_{s} x^{p^{n_{s}}} ;
$$

any $\alpha_{i}$ and $n_{j}$ may occur in the right-hand side (3) for an appropriate $f$. One of the roots of $f$ is 0 . Now take $f$ with $n_{s} \geqslant 1$. By (3) the polynomial $(f+b x) / x$ has degree $p^{n_{s}}-1 \geqslant 1$ and does not vanish at 0 if $b \neq 0$ and $b \neq-\alpha_{1}$. Whence if these inequalities hold, the $G$-stabilizer of $(a, b)$ is nontrivial. Therefore, the action is not locally free.

Moreover, if even the action of $C_{d}$ on $X$ is locally free, and hence $\gamma$ is bijective over a dense open subset of $X$, it may happen that $\gamma$ is purely inseparable. The corresponding example is similar to Example 1.

Example 3 Let char $k=p>0$. Consider the locally free action of $G=B=k^{+}$on $X=k$, given by $b \cdot x=b^{p}+x$. Then $n=1, d=0, C_{0}=G, X_{0}$ is a point, $C_{0} \times X_{0}$ is naturally identified with $G$, and if $\sigma$ maps $X_{0}$ to 0 , then $\gamma^{*}(k(X))=k\left(t^{p}\right) \varsubsetneqq k(t)=$ $k(G)$, where $t$ is the standard coordinate function on $G$. Thus $\gamma$ is not a birational isomorphism. 
4. Below we shall give a characteristic free proof of Theorem 1. For this, we need the following characterization of connected linear algebraic groups $G$ with the property that every rational action of $G$ on an irreducible algebraic variety is birationally equivalent to a regular action of $G$ on an affine algebraic variety.

Definition We say that a linear algebraic group $G$ has property (A) if for every rational action of $G$ on an irreducible algebraic variety $X$, there exist an irreducible affine algebraic variety $Y$ and a birational isomorphism

$$
\varphi: X \rightarrow Y
$$

such that the rational action of $G$ on $Y$ induced by $\varphi$ is regular.

Theorem 2 Let $G$ be a linear algebraic group and let $G^{0}$ be the connected component of the identity in $G$.

(i) If $G^{0}$ is solvable, then $G$ has property (A).

(ii) If $G$ is connected and has property (A), then $G$ is solvable.

Proof (i) Let $G^{0}$ be solvable. Consider a rational action of $G$ on an irreducible algebraic variety $X$. By [8, Theorem 1], there exists an irreducible algebraic variety $X_{1}$ and a birational isomorphism

$$
\alpha_{1}: X \rightarrow X_{1}
$$

such that the rational action of $G$ on $X_{1}$ induced by $\alpha_{1}$ is regular.

Let $v: X_{2} \rightarrow X_{1}$ be the normalization of $X_{1}$. Then the rational action of $G$ on $X_{2}$ induced by the birational isomorphism

$$
\alpha_{2}=v^{-1}: X_{1} \rightarrow X_{2}
$$

is regular, see [3, Theorem 2.25].

By [12, Lemma 8], since $X_{2}$ is a normal algebraic variety, it contains a nonempty $G^{0}$ stable quasi-projective open subset $U$. Hence, by [12, Theorem 1], for some positive integer $n$, there exist a regular action of $G^{0}$ on the projective space $\mathbf{P}^{n}$ and a $G^{0}$ equivariant embedding of the algebraic variety $U$ into $\mathbf{P}^{n}$,

$$
\iota: U \hookrightarrow \mathbf{P}^{n}
$$

We may (and shall) assume that $n$ is minimal possible with this property. Since Aut $\mathbf{P}^{n}=\mathrm{PGL}_{n}$, this action of $G^{0}$ on $\mathbf{P}^{n}$ induces an action of $G^{0}$ on the dual projective space $\check{\mathbf{P}}^{n}$. By the Borel fixed-point theorem [11, Theorem 6.2.6], the assumption that $G^{0}$ is a connected solvable linear algebraic group implies that in $\check{\mathbf{P}}^{n}$ there is a fixed point of this action. This means that $\mathbf{P}^{n}$ contains a $G^{0}$-stable hyperplane $H$. Hence $\mathbf{P}^{n} \backslash H$ is a $G^{0}$-stable affine open subset of $\mathbf{P}^{n}$. Therefore, the minimality assumption on $n$ implies that $\iota(U) \cap\left(\mathbf{P}^{n} \backslash H\right)$ is a nonempty $G^{0}$-stable open quasiaffine subset of $\iota(U)$. This proves that $X_{2}$ contains a nonempty $G^{0}$-stable open quasiaffine subset $V$. 
Normality of $G^{0}$ in $G$ then implies that $g \cdot V$ for every element $g \in G$ is a nonempty $G^{0}$-stable open quasiaffine subset of $X_{2}$.

By [1, Lemma 5.11 and the footnote to its proof], there exists a finite subgroup $F$ of $G$ that intersects every connected component of $G$. Put

$$
X_{3}=\bigcap_{g \in F} g \cdot V .
$$

Then $X_{3}$ is $G^{0}$ - and $F$-stable and, therefore, $G$-stable. Let

$$
\alpha_{3}: X_{2}-\rightarrow X_{3}
$$

be the birational isomorphism inverse to the identity embedding $X_{3} \hookrightarrow X_{2}$. Thus we have proved that $X_{3}$ is a quiasiaffine algebraic variety such that the rational action of $G$ on $X_{3}$ induced by $\alpha_{3}$ is regular.

Finally, by [9, Lemma 2] (see also [7, Theorem 1.6]) quasiaffiness of $X_{3}$ implies that there exist an irreducible affine algebraic variety $X_{4}$ endowed with a regular action of $G$ and a $G$-equivariant birational embedding

$$
\alpha_{4}: X_{3} \hookrightarrow X_{4}
$$

This shows that we may take $Y=X_{4}$ and $\varphi=\alpha_{4} \circ \alpha_{3} \circ \alpha_{2} \circ \alpha_{1}$.

(ii) Let the group $G$ be connected and has property (A). Assume that it is non-solvable. Then it contains a proper parabolic subgroup $P$; see [11, Proposition 6.2.5]. Let $X$ be $G / P$ endowed with the natural action of $G$. We have $\operatorname{dim} X>0$. Let $Y$ and $\varphi$ be respectively an irreducible affine algebraic variety endowed with a regular action of $G$ and a birational isomorphism (4), whose existence is ensured by property (A). Since $\varphi$ is $G$-equivariant and the action of $G$ on $X$ is transitive, $\varphi$ is a morphism. Therefore, completeness and irreducibility of $X$ implies that $\varphi(X)$ is a complete $G$-stable closed irreducible subset in $Y$; see [11, Proposition 6.1.2(iii)]. Since $Y$ is affine, this yields that $\varphi(X)$ is a point; see [11, Proposition 6.1.2(vi)]. But $\operatorname{dim} \varphi(X)=\operatorname{dim} X>0$ because $\varphi$ is a birational isomorphism-a contradiction.

5. We also need the following statement, see [11, Proposition 14.2.2] and an earlier result [5, Lemma 1.5].

Proposition 1 Let $G=k^{+}$and let $X$ be an irreducible affine algebraic variety endowed with a nontrivial regular action of $G$. Then there exists an irreducible affine variety $Y$ with the following properties:

(a) there is an isomorphism $\phi$ of $G \times Y$ onto an open subvariety of $X$;

(b) there is a morphism $\psi: G \times Y$ such that for all $a, b \in k, y \in Y$,

$$
\psi(a+b, y)=\psi(a, y)+\psi(b, y), \quad a \cdot \phi(b, y)=\phi(\psi(a, y)+b, y) .
$$


Corollary Maintain the notation of Proposition 1. Then the formula

$$
a \cdot(b, y)=(\psi(a, y)+b, y), \quad a, b \in G, \quad y \in Y,
$$

defines a regular action of $G$ on $G \times Y$ such that

- the natural projection $\mathrm{pr}_{2}: G \times Y \rightarrow Y$ is its rational quotient;

- the isomorphism $\phi$ is G-equivariant.

In particular, $Y$ and $X, i G$ are birationally isomorphic.

6. We now turn to a characteristic free proof of Theorem 1 .

Proof (Characteristic free proof of Theorem 1) We retain the argument in [4], except its part referring to the cross-section theorem of [8] that works, as we have explained, only if char $k=0$. This part is replaced by the following characteristic free argument.

By Theorem 2, we may assume that $X$ is affine and the action of the one-dimensional connected linear algebraic group $H=B_{d} / B_{d+1}$ on $X$ is nontrivial and regular. There are two possibilities: $H$ is isomorphic to either $k^{+}$or $k^{\times}$.

Let $H$ be isomorphic to $k^{+}$. Then by Corollary of Proposition 1 the variety $X$ is $H$-equivariantly birationally isomorphic to the variety $\mathbf{P}^{1} \times X_{d}$, on which $H$ acts rationally so that the second projection $\mathrm{pr}_{2}: \mathbf{P}^{1} \times X_{d} \rightarrow X_{d}$ is a rational quotient of this action.

It remains to show that the same is true if $H$ is isomorphic to $k^{\times}$. The group $X(H)$ of characters of $H$ (i.e., algebraic homomorphisms $H \rightarrow k^{\times}$) is isomorphic to $\mathbf{Z}$. Let $\chi$ be its generator. For every $s \in \mathbf{Z}$, put

$$
k(X)_{s}=\left\{f \in k(X): h \cdot f=\chi^{s}(h) f \text { for every } h \in H\right\}
$$

in particular, $k(X)_{0}=k(X)^{H}$. Since $H$ is a torus, the $H$-module $k[X]$ is semisimple and its isotypic decomposition has the form

$$
k[X]=\bigoplus_{s \in \mathbf{Z}} k[X]_{s}, \quad \text { where } k[X]_{s}=k(X)_{s} \cap k[X]
$$

(see, e.g., $[11,3.2 .13]$ ); in particular, $k[X]_{0}=k[X]^{H}$. Given (6), every element $f \in$ $k[X]$ can be uniquely written as the following sum of the nonzero summands:

$$
f=f_{i_{1}}+\cdots+f_{i_{s}}, \quad f_{j} \in k[X]_{j} \text { for all } j .
$$

We call (7) the canonical decomposition of $f$. Nontriviality of the action of $H$ on $X$ implies that $k[X]^{H} \neq k[X]$. Therefore, the subgroup

$$
\Gamma=\left\{s \in \mathbf{Z}: k(X)_{s} \neq 0\right\}
$$

of $\mathbf{Z}$ is nonzero, i.e., $\Gamma=n \mathbf{Z}$ for some positive integer $n$. 
It follows from (8) that there are $a_{1}, \ldots, a_{m} \in \mathbf{Z}$ such that in canonical decomposition (7) we have

$$
i_{1}=n a_{1}, \quad \ldots, \quad i_{s}=n a_{s} .
$$

Fix a nonzero element $t \in k(X)_{n}$. From (5), (7), and (9) we deduce that

$$
f_{i_{r}} / t^{a_{r}} \in k(X)^{G} \quad \text { for every } r
$$

In turn, (7) and (10) yield that $f=\sum_{r=1}^{s} t^{a_{r}}\left(f_{i_{r}} / t^{a_{r}}\right)$ is an element of the subfield $k(X)^{H}(t)$ of $X$. Hence $k[X]$ lies in this subfield. But $k(X)$ is the field of fractions of $k[X]$ because $X$ is affine. This proves that

$$
k(X)=k(X)^{H}(t) .
$$

The element $t$ is transcendental over $k(X)^{H}$. Indeed, if not, there is a relation $\sum_{i=0}^{m} a_{i} t^{r_{i}}=0$ for some integers $0 \leqslant r_{0}<r_{1}<\cdots<r_{m}$ and nonzero elements $a_{i} \in k(X)^{H}$. From (5) we then deduce that

$$
\sum_{i=0}^{m} \chi^{n r_{i}}(h) a_{i} t^{r_{i}}=0 \quad \text { for every element } h \in H
$$

This contradicts Artin's theorem on independence of characters, because $k^{\times}$is the subgroup of the multiplicative group $k(X)^{\times}$of $k(X)$, and therefore, we may consider the elements of $\mathcal{X}(H)$ as the homomorphisms $H \rightarrow k(X)^{\times}$.

Given that $t$ is transcendental over $k(X)^{H}$, we conclude from (11) that $X$ is $H$ equivariantly birationally isomorphic to the variety $\mathbf{P}^{1} \times X_{i}^{\prime} H$, on which $H$ acts rationally via the first factor so that the second projection $\mathrm{pr}_{2}: \mathbf{P}^{1} \times X_{\prime}^{\prime} H \rightarrow X_{\prime}^{\prime} H$ is a rational quotient of this action. This completes the proof.

7. Combining the given proof of Theorem 1 with Rosenlicht's theorem on the existence of generic geometric quotient [10, Theorem], we obtain the following generalization of the result of [2, Section 1] on "trivial quotient" (our attention was drawn to this result by G. Kemper, whom we thank).

Theorem 3 Let $X$ be an irreducible algebraic variety endowed with a regular action of a solvable connected linear algebraic group $G$. Then for the restriction of this action on a certain $G$-stable dense open subset $U$ of $X$ there exist

- the geometric quotient $\pi_{G, U}: U \rightarrow U / G$;

- an isomorphism $\varphi: U \rightarrow \mathbf{A}^{r, s} \times(U / G)$, where

$$
\mathbf{A}^{r, s}=\left\{\left(\alpha_{1}, \ldots, \alpha_{r+s}\right) \in \mathbf{A}^{r+s}: \alpha_{i} \neq 0 \text { for every } i \leqslant r\right\}, \quad r \geqslant 0, \quad s \geqslant 0,
$$

such that the natural projection $\mathbf{A}^{r, s} \times(U / G) \rightarrow U / G$ is the geometric quotient of the regular action of $G$ on $\mathbf{A}^{r, s} \times(U / G)$ induced by $\varphi$.

Theorem 3 immediately implies the cross-section theorem. 
Corollary ([8, Theorem 10]) Let $X$ be an irreducible algebraic variety endowed with a regular action of a solvable connected linear algebraic group $G$. Let $\pi_{G, X}: X \rightarrow$ $X ; G$ be a rational quotient of this action. Then there is a rational map $\sigma: X ; G \rightarrow X$ such that $\pi_{G, X} \circ \sigma=\mathrm{id}$.

Acknowledgment The author is indebted to the referee for thorough reading and remarks.

\section{References}

1. Borel, A., Serre, J.-P.: Théorèmes de finitude en cohomologie galoisienne. Comment. Math. Helv. 39, 111-164 (1964)

2. Greuel, G.-M., Pfister, G.: Geometric quotients of unipotent group actions. Proc. Lond. Math. Soc. 67(1), 75-105 (1993)

3. Iitaka, S.: Algebraic Geometry. Graduate Texts in Mathematics, vol. 76. North-Holland Mathematical Library, vol. 24. Springer, New York (1982)

4. Matsumura, H.: On algebraic groups of birational transformations. Atti Accad. Naz. Lincei Rend. Cl. Sci. Fis. Mat. Natur 34, 151-155 (1963)

5. Miyanishi, M.: Lectures on Curves on Rational and Unirational Surfaces. Tata Institute of Fundamental Research Lectures on Mathematics, vol. 60. Narosa, New Delhi (1978)

6. Popov, V.L.: Some subgroups of the Cremona groups. In: Masuda, K., Kojima, H., Kishimoto, T. (eds.) Affine Algebraic Geometry (Osaka, 2011), pp. 213-242. World Scientific, Singapore (2013)

7. Popov, V.L., Vinberg, E.B.: Invariant theory. In: Parshin, A.N., Shafarevich, I.R. (eds.) Algebraic Geometry IV. Encyclopaedia of Mathematical Sciences, vol. 55, pp. 123-284. Springer, Berlin (1994)

8. Rosenlicht, M.: Some basic theorems on algebraic groups. Amer. J. Math. 78(2), 401-443 (1956)

9. Rosenlicht, M.: On quotient varieties and the affine embedding of certain homogeneous spaces. Trans. Amer. Math. Soc. 101(2), 211-223 (1961)

10. Rosenlicht, M.: A remark on quotient spaces. An. Acad. Brasil. Ciênc. 35, 487-489 (1963)

11. Springer, T.A.: Linear Algebraic Groups. Progress in Mathematics, vol. 9, 2nd edn. Birkhäuser, Boston (1998)

12. Sumihiro, H.: Equivariant completion. J. Math. Kyoto Univ. 14(1), 1-28 (1974) 